\title{
IRVE-II Post-Flight Trajectory Reconstruction
}

\author{
Stephen A. O'Keefe ${ }^{1}$ and David M. Bose ${ }^{2}$ \\ Analytical Mechanics Associates, Inc, Hampton, VA 23666
}

\begin{abstract}
NASA's Inflatable Re-entry Vehicle Experiment (IRVE) II successfully demonstrated an inflatable aerodynamic decelerator after being launched aboard a sounding rocket from Wallops Flight Facility (WFF). Preliminary day of flight data compared well with pre-flight Monte Carlo analysis, and a more complete trajectory reconstruction performed with an Extended Kalman Filter (EKF) approach followed. The reconstructed trajectory and comparisons to an attitude solution provided by NASA Sounding Rocket Operations Contract (NSROC) personnel at WFF are presented. Additional comparisons are made between the reconstructed trajectory and pre and post-flight Monte Carlo trajectory predictions. Alternative observations of the trajectory are summarized which leverage flight accelerometer measurements, the pre-flight aerodynamic database, and on-board flight video. Finally, analysis of the payload separation and aeroshell deployment events are presented. The flight trajectory is reconstructed to fidelity sufficient to assess overall project objectives related to flight dynamics and overall, IRVE-II flight dynamics are in line with expectations.
\end{abstract}

\section{Nomenclature}

$\begin{array}{ll}\alpha_{\text {total }} & =\text { Total angle of attack, degrees } \\ C_{A} & =\text { Axial force coefficient } \\ C_{N} & =\text { Normal force coefficient } \\ C_{Y} & =\text { Side force coefficient } \\ C_{m} & =\text { Pitching moment coefficient } \\ C_{w} & =\text { Yawing moment coefficient } \\ C_{l} & =\text { Rolling moment coefficient }\end{array}$

\section{Introduction}

N August 17, 2009 NASA Langley Research Center's Inflatable Re-entry Vehicle Experiment II (IRVE-II) successfully demonstrated the use of an inflatable aerodynamic decelerator. IRVE-II is a build-to-print re-flight of the IRVE-I mission ${ }^{1}$ launched in 2007. While IRVE-I suffered a flight mishap while separating from the sounding rocket, IRVE-II was a resounding success. IRVE-II was launched aboard a Terrier-Black Brant IX sounding rocket from NASA's Wallops Flight Facility (WFF). Spin stabilized at $3.5 \mathrm{~Hz}$ the inflatable aeroshell was deployed exo-atmospheric and entered the atmosphere unguided. The following details the post-flight reconstruction analysis performed for this mission.

While data was collected for the entire trajectory, the IRVE-II experiment was defined between $80 \mathrm{~km}$ and $40 \mathrm{~km}$ altitude. Therefore, an inflated view of this region between 416 seconds and 446 seconds after launch is provided. Pre-flight trajectory analysis and predictions used $2 \sigma$ bounds; as such, the bounds of simulated Monte Carlo results are included in many of the following plots. Note that the 97.72 percentile denoted in the plots is equivalent to two standard deviations past the mean for a Gaussian distribution, the probability that a value is within \pm 2 standard deviations of the mean is 95.45 , the number also commonly associated with " $2 \sigma$ ". The 99.87 percentile is equivalent to three standard deviations past the mean.

\section{Quick-Look Trajectory}

Quick-look trajectory data was provided by WFF several hours after the launch of IRVE-II. This data included altitude, horizontal range, and velocity from time of launch until 1093.5 seconds into flight. As a preliminary

\footnotetext{
${ }^{1}$ Project Engineer

${ }^{2}$ Vice President, Analytical Mechanics Associates, Inc, AIAA Member 


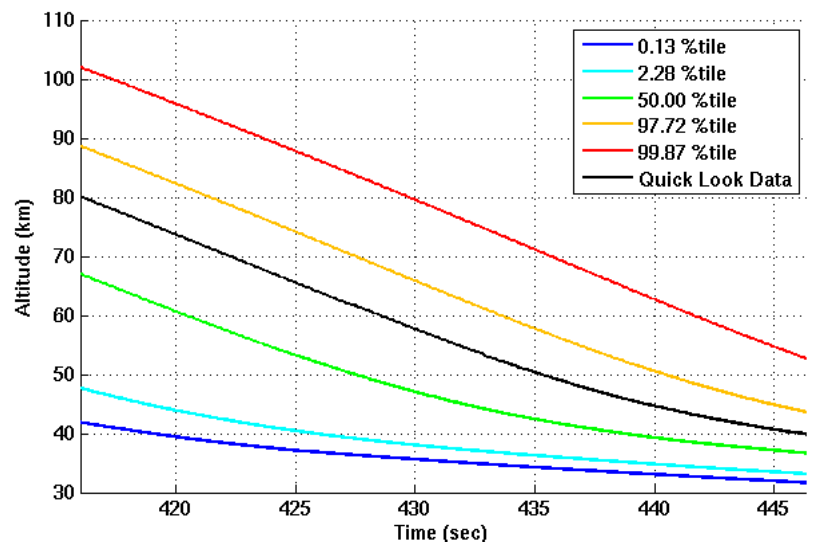

(a)

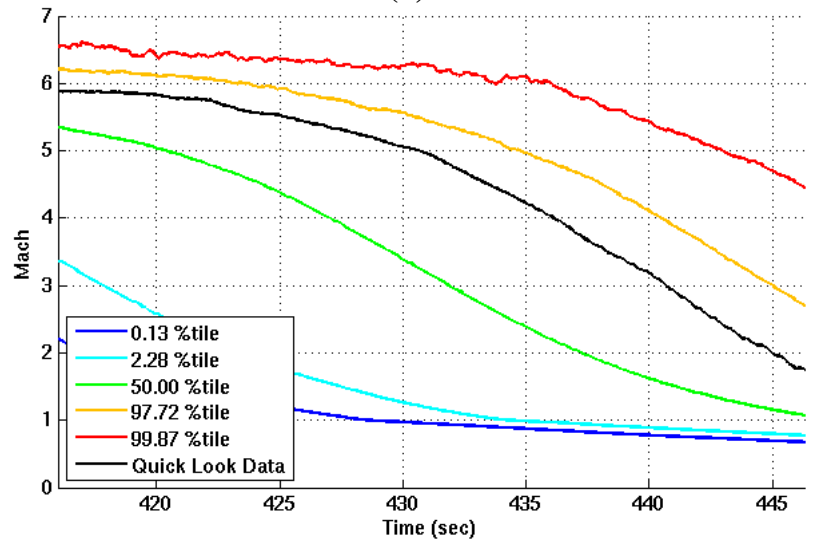

(c)

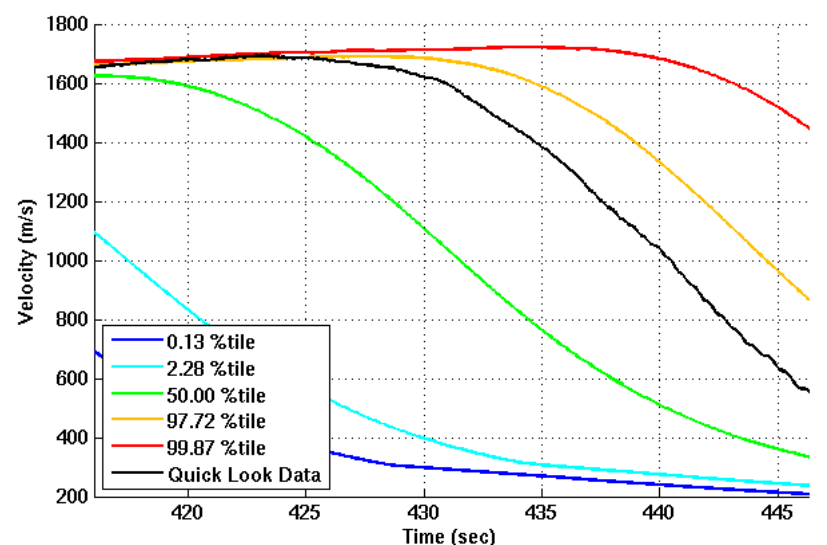

(b)

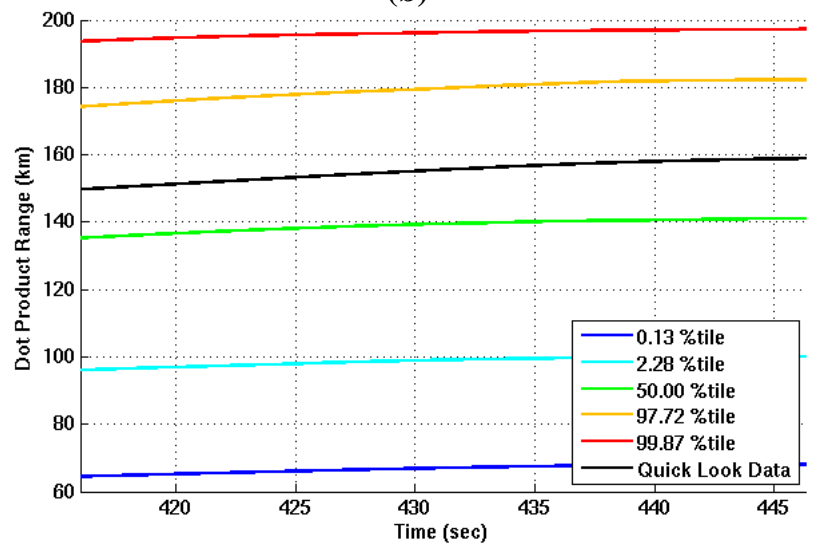

(d)

Figure 1. Quick-look data compared to pre-flight Monte Carlo bounds.

evaluation of the performance of IRVE-II, the quick-look trajectory is compared to the pre-flight simulation Monte Carlo results. The velocity included in the quick-look data is combined with the nominal GRAM07 WFF Range Reference Atmosphere (RRA) for the month of August to give an estimate of Mach. Plots of altitude, velocity, Mach number and downrange distance are shown in Figure 1.

During the period of the experiment the quick-look data falls between the 50 and 97.72 percentile bounds of the pre-flight Monte Carlo simulation. As can be seen in Figure 1 (b), IRVE-II separated from the sounding rocket booster at 90 seconds with a higher than predicted nominal velocity. This correlates well with the higher than nominal altitude and downrange also seen and is a preliminary indication that IRVE-II performed within the predicted $2 \sigma$ trajectory bounds.

\section{Trajectory Reconstruction}

In order to assess the performance of IRVE-II, data from the flight is used to reconstruct a Best Estimate Trajectory (BET). The reconstruction approach leverages several data sources including on board instrumentation, ground radar data and atmospheric measurements blended through the application of an Extended Kalman Filter (EKF) using the NewSTEP tool. The NewSTEP tool, data sources and their associated uncertainties, and data preprocessing are examined in detail in the following section.

\section{A. Approach}

The goal of the reconstruction is to determine the best estimated trajectory representing the vehicle state time history that most accurately fits all available measurement data. To achieve this, the NewSTEP Trajectory Reconstruction tool is used, which centers on an Extended Kalman Filter. NewSTEP is an Analytical Mechanics Associates, Inc. developed tool based on the numerical approach used in the Statistical Trajectory Estimation Program (STEP). STEP was used extensively in the 1960s and 1980s on projects including X-23A PRIME, Viking, Pioneer Venus and Shuttle. NewSTEP adds many enhancements to the original STEP tool including migration to 
MATLAB based code, addition of measurements and updated filters. It has been used in recent missions such as the Mars Exploration Rover (MER) and X-43A (Hyper-X) with ongoing work on Ares I-X, Orion Pad Abort 1 and Mars Science Laboratory (MSL) Entry Air Data System (MEADS). "Hyper-X Post Flight Trajectory Reconstruction" by Karlgaard $^{2}$, et.al, provides a detailed discussion of the NewSTEP tool, its filters, and its numerical methods.

A similar reconstruction effort has been undertaken by the NASA Sounding Rocket Operations Contract (NSROC) team. Their effort centers on the Algebraic Method, which infers attitude and roll rate based on solar sensor and magnetometer measurements. While not discussed in detail here, the NSROC solution was expected to produce an attitude solution for IRVE-II up to deployment of the inflatable with no guarantee of good data beyond this point. However, the NSROC team has been successful in producing a good solution well into re-entry including the region of the experiment. As a result, their solution is used both to initialize the NewSTEP reconstruction and to cross-validate results.

\section{B. Data Sources \& Key Assumptions}

NewSTEP is capable of blending together data from multiple sources. For IRVE-II these data sources include multiple ground radar sites, on-board accelerometers and rate sensors and atmospheric measurements provided by the WFF range. Several WFF ground radar sites tracked IRVE-II via C-band transponders. IRVE-II was equipped with three on-board Systron Donner BEI QRS 11 rate sensors and a NSROC(a) containing 3-axis Setra accelerometers. Mass properties were measured pre-flight and atmosphere data was provide by several sources, including high altitude environments from a VIPER meteorological rocket, and low altitude winds from balloon data.

Four radar stations tracked IRVE-II during flight, designated Radars 2, 3, 5, and 18. Radars 5 and 18 show a good track of IRVE-II and are used in the reconstruction effort. Radar 2 data includes significant noise with amplitudes well beyond the expected accuracy of the radar and Radar 3 exhibits a positional offset that does not correspond to the other radar tracks. Therefore, these two radar tracks have been excluded from the reconstruction.

Prior to use in NewSTEP the raw rate sensor and accelerometer data is converted from counts to engineering units and rotated from the sensor coordinate frame to a common body frame. This frame is defined with the x-axis pointing along the axis of symmetry through the nose of IRVE-II, the y-axis pointing through the pyrotechnic cutter block and the z-axis completing the right handed coordinate system. In addition to the measured data, NewSTEP requires initial estimates of the uncertainty in sensor location, misalignment, noise, scale factor and bias for the rate sensors and accelerometers, which come from a combination of pre-flight measurements and calibrations as well as estimates based on engineering judgment.

Mass properties used in NewSTEP are derived from pre-flight measurements. The flight is divided into two sets of values, those before (stowed) and those after inflation (deployed). As opposed to the stowed values, the deployed configuration values were not directly measured. Results of the stowed configuration measurements were used to calibrate a 3D CAD model which was used to extract mass properties for the deployed configuration.

Atmosphere data used in NewSTEP comes from several different sources including a meteorological rocket, weather balloons and a reference atmosphere model. One hour and thirty six minutes after the launch of IRVE-II, a VIPER rocket was launched from WFF to measure meteorological conditions in the upper atmosphere. The VIPER rocket returned data for density, pressure, temperature, north and east winds and their corresponding RMS values between $30.5 \mathrm{~km}$ and $92 \mathrm{~km}$ altitude. No adjustment is made for the difference in launch times between the VIPER and IRVE-II, and the data from the VIPER are assumed to represent steady state values. Weather balloons released from WFF at the time of IRVE-II launch provide wind data for the lower atmosphere. North and east wind velocities were measured for the altitude range of $122 \mathrm{~m}$ to $36.6 \mathrm{~km}$. No adjustment is made for time and location differences relative to the IRVE-II trajectory. For this altitude range, and all others not covered by the VIPER data, uncertainties are generated by Earth GRAM07 using the August WFF RRA.

The NewSTEP reconstruction is initialized at 295 seconds after launch from conditions supplied by the NSROC reconstruction solution, Radar 5 and the reference atmosphere model. Data from the accelerometers and rate sensors are available prior to this time; however, the roll rate sensor is saturated prior to 295 seconds and signal to noise ratio on all acceleration measurements is very poor. Position and velocity are computed from Radar 5 data and attitude from the NSROC solution. Atmosphere values are estimated using the mean and standard deviation values from Earth GRAM07.

\section{Preprocessing}

Raw sensor data must be preprocessed before being used in NewSTEP. Data from the accelerometers and rate sensors was received at $1250 \mathrm{~Hz}$ and preprocessed prior to use in NewSTEP. This preprocessing includes removal of rogue points, interpolation to a constant rate, smoothing and filtering. Rogue points are removed using evaluation of 


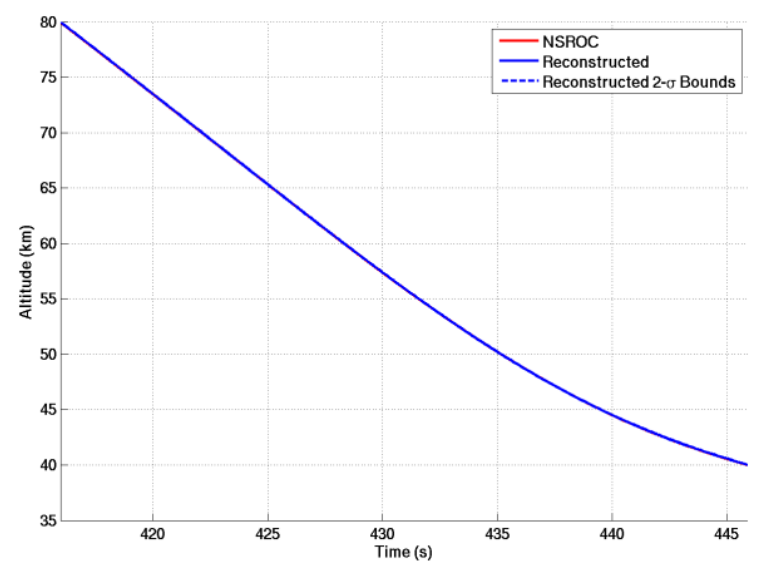

(a)

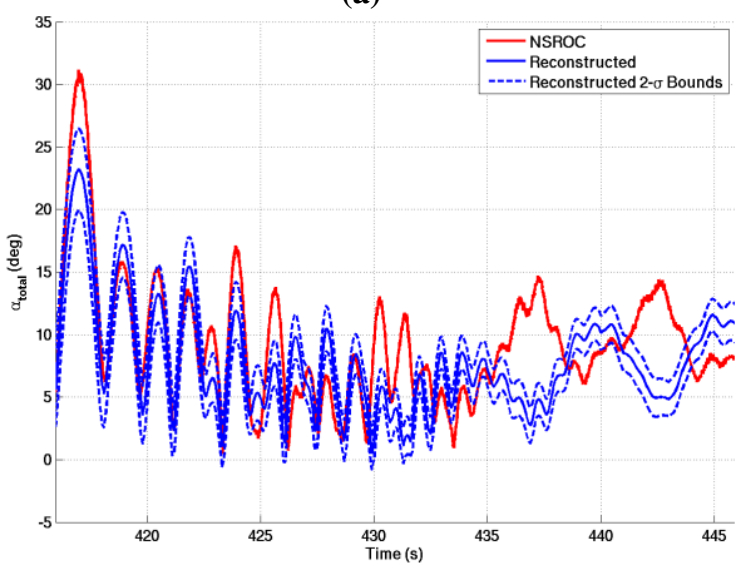

(c)

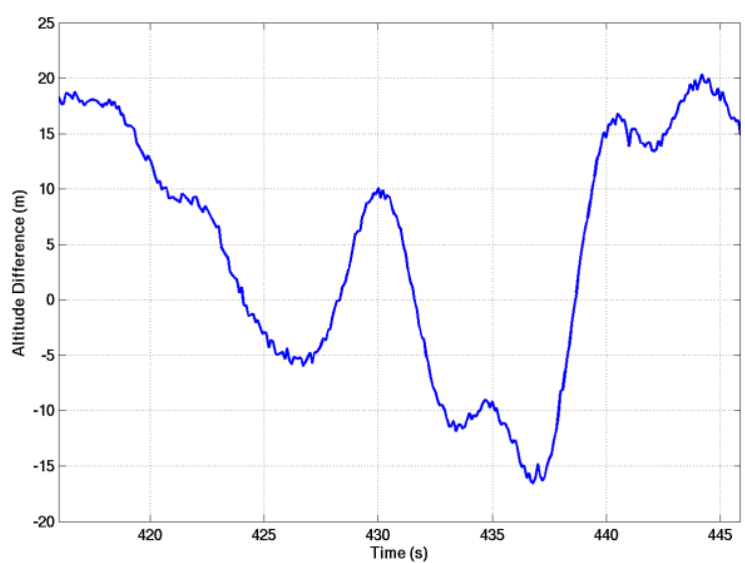

(b)

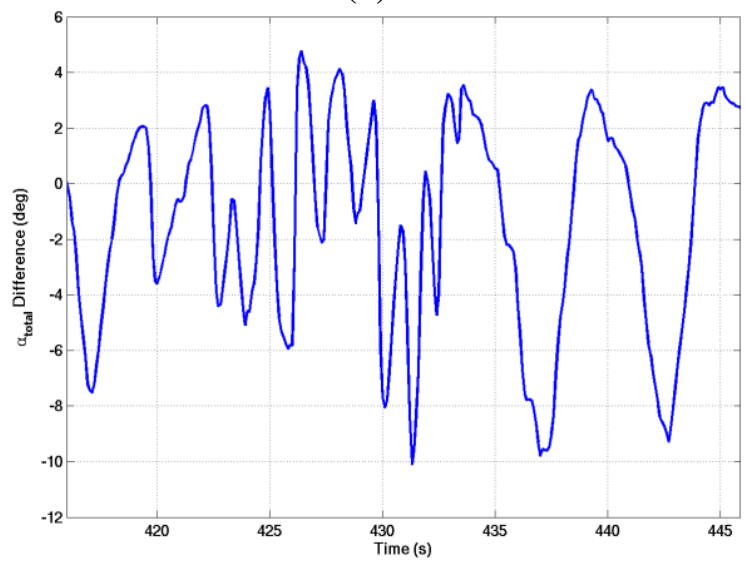

(d)

Figure 2. Comparison of NSROC and NewSTEP reconstruction results.

the rate of change of the signal and engineering judgment. The data is then linearly interpolated at a constant rate of $60 \mathrm{~Hz}$. Next, smoothed time histories and noise covariance matrix estimates are computed using optimal Fourier smoothing. Finally, a low pass filter is applied in the frequency domain, in order to introduce no phase loss, using a $5 \mathrm{~Hz}$ cut-off frequency. The $5 \mathrm{~Hz}$ cut-off frequency is well above the $1.5 \mathrm{~Hz}$ expected maximum rigid body frequencies for flight, resulting in negligible gain loss at the frequencies of interest.

\section{Reconstruction Results}

Pre-flight expectations for the trajectory reconstruction accuracy were modest. Due to the fidelity of on-board instrumentation, $2 \sigma$ attitudes within 6 degrees during peak pressure were anticipated. Allowing for the fidelity of measurements involved, the reconstruction performed favorably.

Comparisons are made to several independent sources to ensure the validity of the NewSTEP results. In this section the results of the NewSTEP reconstruction are compared to the NSROC solution as well as pre- and postflight Monte Carlo simulation bounds. In addition, the reconstructed aerodynamic coefficients are compared to values predicted by the pre-flight aerodynamic database.

\section{A. Comparison to NSROC Solution}

The independent NSROC solution proved more viable than originally expected during re-entry and the results are used here to cross-validate the NewSTEP solution. Figure 2 presents comparisons between the NSROC and NewSTEP reconstruction results for altitude, total angle of attack and roll rate. Calculated differences between the two results are also shown as a function of time.

Overall the results from the NewSTEP reconstruction compare favorably with the results from the NSROC reconstruction. Both solutions compare well in altitude, within $20 \mathrm{~m}$, and show similar trends and magnitudes in 
total angle of attack. While differences between the two results do exceed the anticipated 6 degrees, they remain within 10 degrees. Prior to 435 seconds the NSROC and NewSTEP solutions match well in frequency if not in magnitude. After 435 seconds, there is an inversion of the oscillations between the two angle of attack solutions. This discrepancy occurs during the entry pressure pulse and is seen in the reconstructed aerodynamic coefficients discussed later.

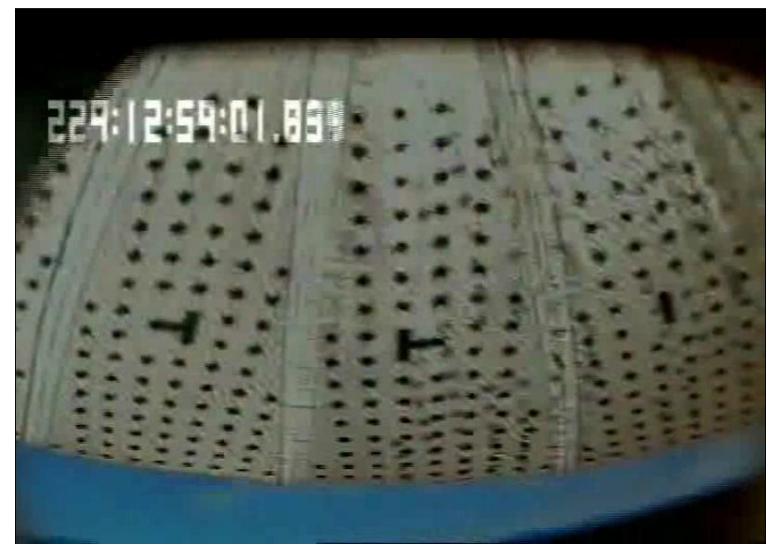

(a)

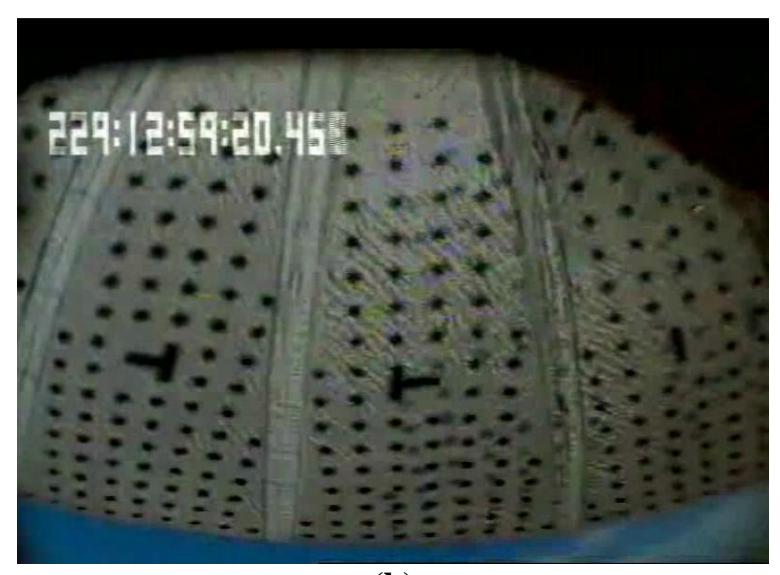

(b)

Figure 3. On-board flight video of the aft side of the aeroshell at 421 seconds (a) and 440 seconds (b).

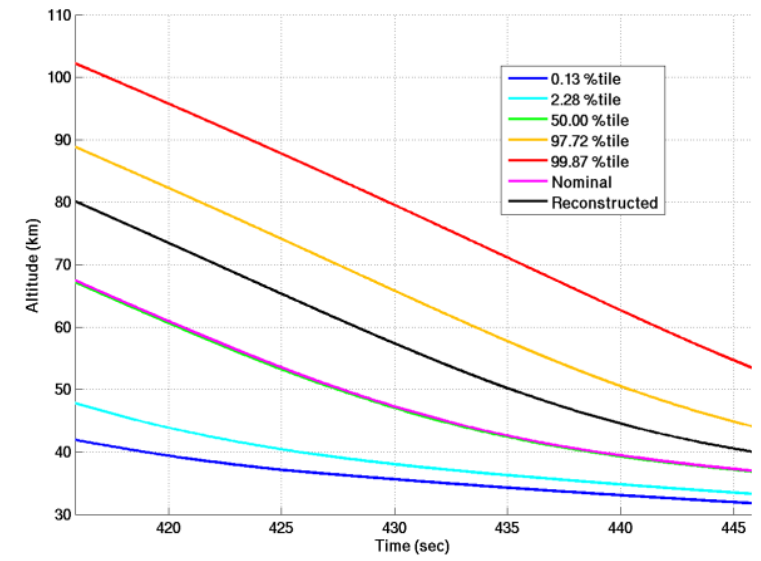

(a)

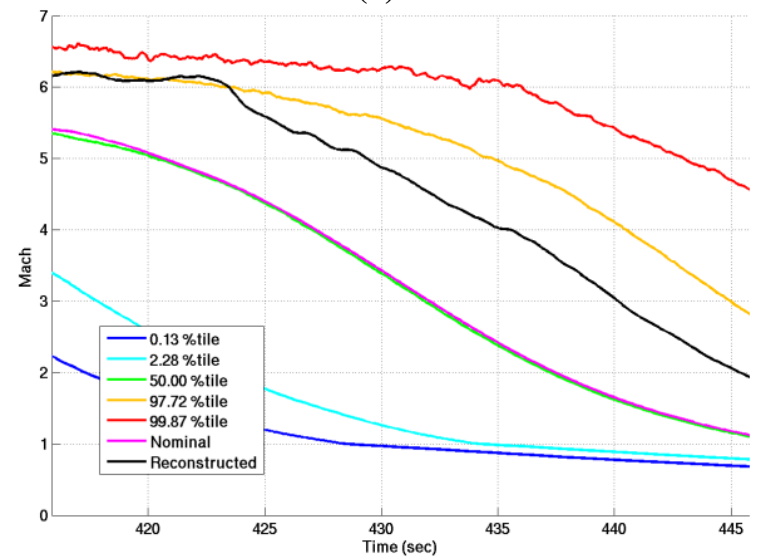

(c)

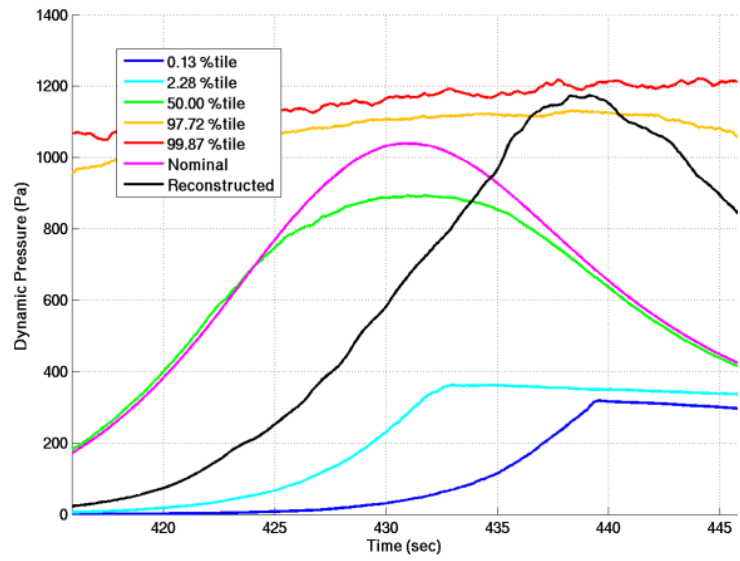

(b)

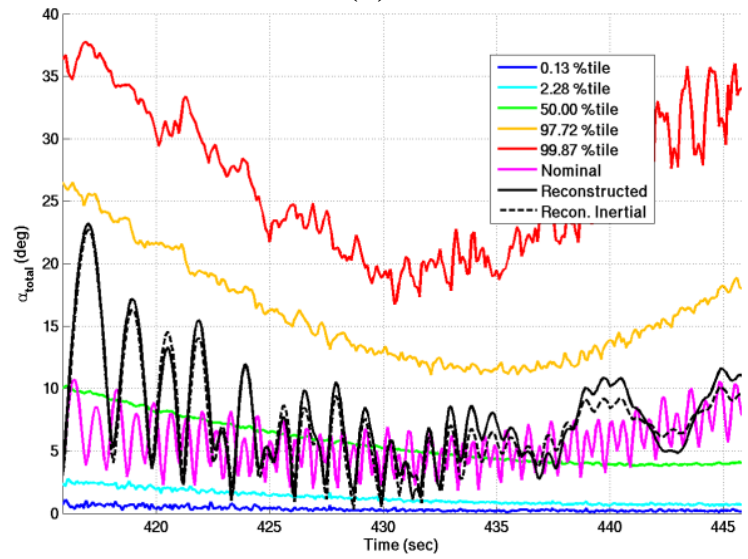

(d)

Figure 4. Calculated trajectory data compared to pre-flight simulated Monte Carlo bounds. 


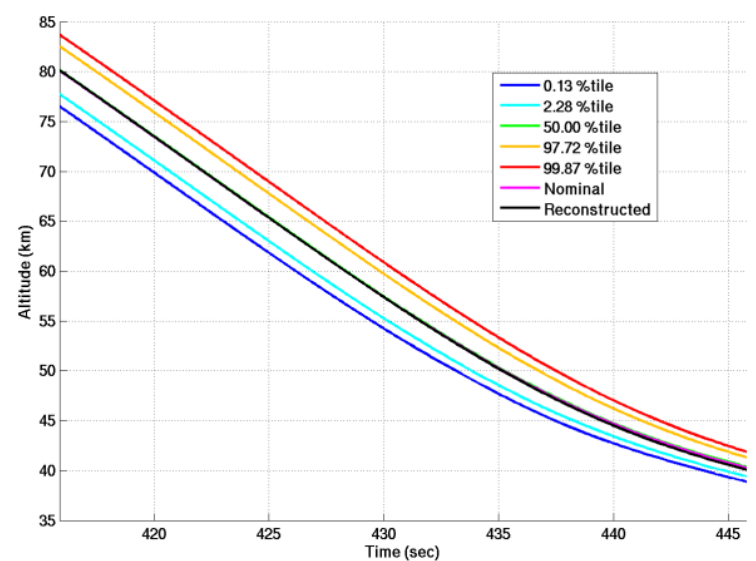

(a)

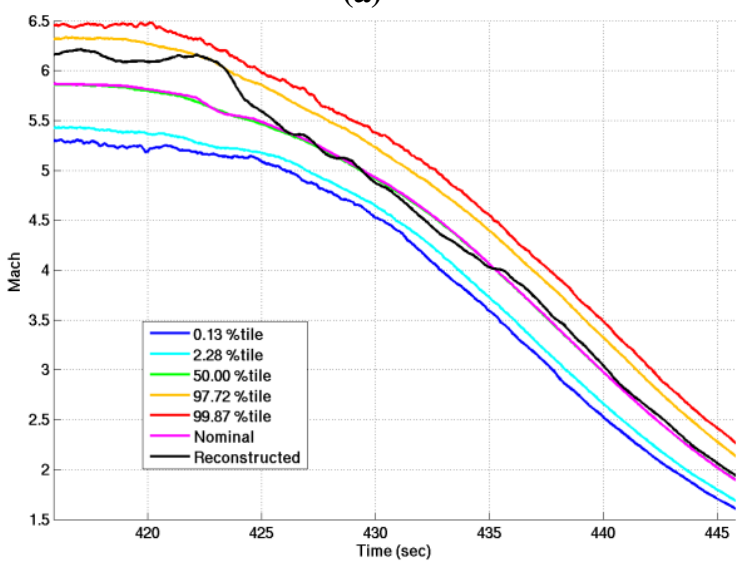

(c)

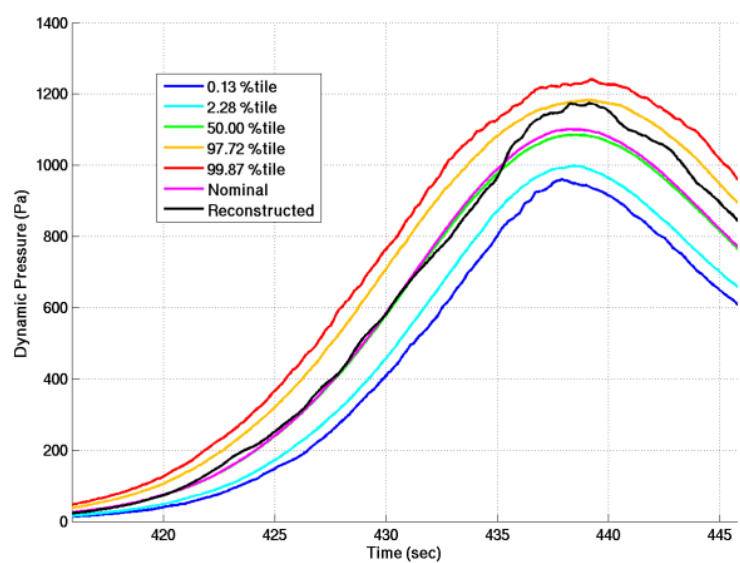

(b)

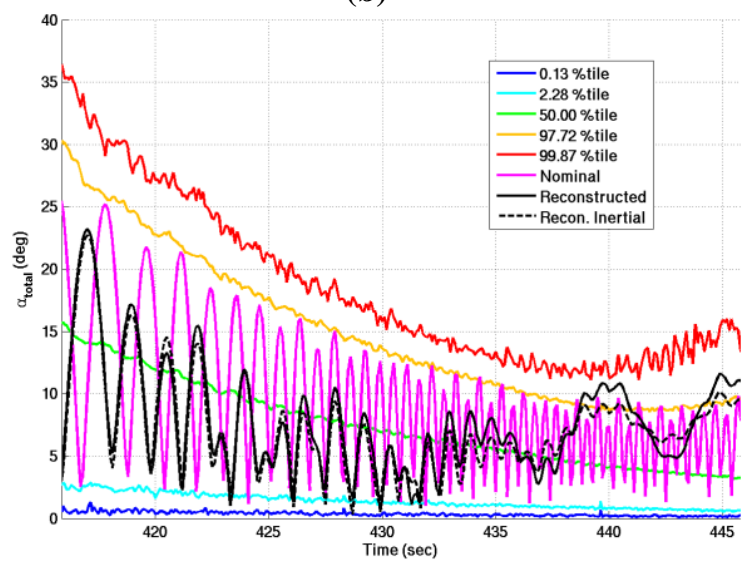

(d)

Figure 5. Reconstructed trajectory data compared to post-flight simulated Monte Carlo bounds.

\section{B. Comparison to Monte Carlo Predictions}

Prior to the flight of IRVE-II a Monte Carlo simulation was developed using the Program to Optimize Simulated Trajectories II (POST2). This simulation takes into account the measured mass properties, predicted trajectory states at separation from the sounding rocket, a pre-flight aerodynamic model and atmospheric variations and calculates expected trajectories. The aerodynamic model was based on both wind tunnel data and CFD analysis assuming a rigid aeroshell and the atmosphere was simulated using Earth GRAM07.

Post-flight the Monte Carlo is rerun updating the timeline and initial conditions with known flight values. The initial conditions at separation, 90 seconds into flight, are taken from the NSROC attitude solution and radar data. The timeline for the aeroshell retention bag release and inflation are updated accordingly. Reconstruction results are plotted against both the pre-flight and post-flight Monte Carlo simulation bounds in the following sections.

While the Monte Carlo simulations assume that the IRVE-II aeroshell remains rigid throughout the entire trajectory, there is evidence that IRVE-II flexed during the dynamic pressure pulse. Figure 3 shows two frames from flight video recorded by a camera mounted on the center-body. On the left is a frame from 421 seconds and on the right is a frame from 440 seconds, roughly corresponding to peak dynamic pressure. In the image on the right the aeroshell appears to occupy more of the field of view, with its edge closer to the bottom of the frame. Since the camera remains rigidly attached to the center-body, this may be due to aeroshell deflection or rotation of the centerbody axis of symmetry relative to the aeroshell axis of symmetry. This effect could change both the alignment of the accelerometers and the aerodynamics of the vehicle, which would explain some discrepancies between the measured accelerations and pre-flight estimates. Further collaboration is required with structural and photogrammetric analysis in order to quantify this effect and its impact on flight measurements. It is important to note that reconstructed trajectories are based on sensors within the center-body. As such, the trajectories represent the state of the centerbody. 


\section{Pre-Flight Monte Carlo Predictions}

The pre-flight Monte Carlo simulation results bound the reconstructed trajectory well. Plots of these bounds and the respective reconstruction values are shown in Figure 4. Altitude and total angle of attack remain within the $2 \sigma$ predictions. Dynamic pressure exceeds the $2 \sigma$ predictions, but remains within $3 \sigma$ predictions. The reconstruction exhibits peak dynamic pressure approximately 8 seconds later than the pre-flight simulation. This timing difference is believed to be caused by a difference in the initial conditions of IRVE-II at separation, and is not evident when the results are compared to the post-flight Monte Carlo.

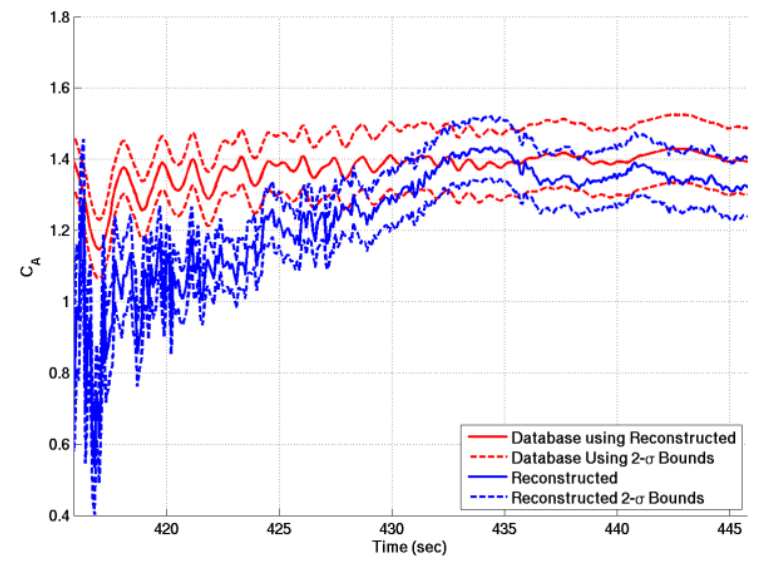

(a)

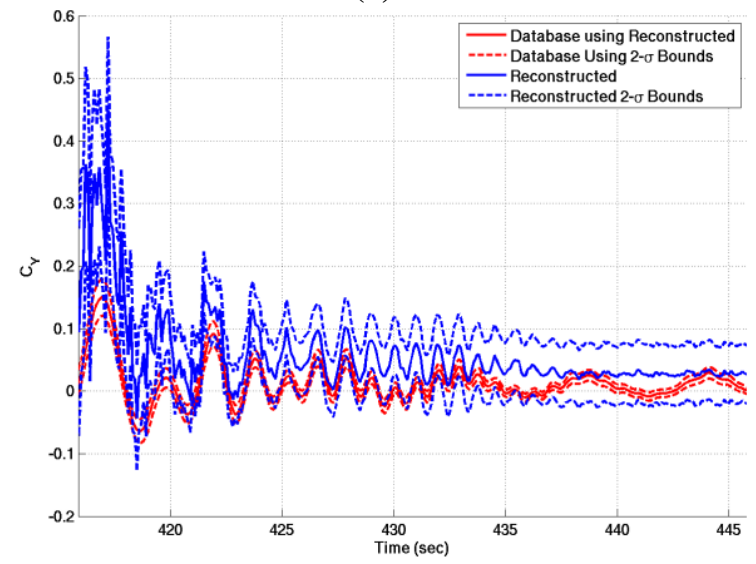

(c)

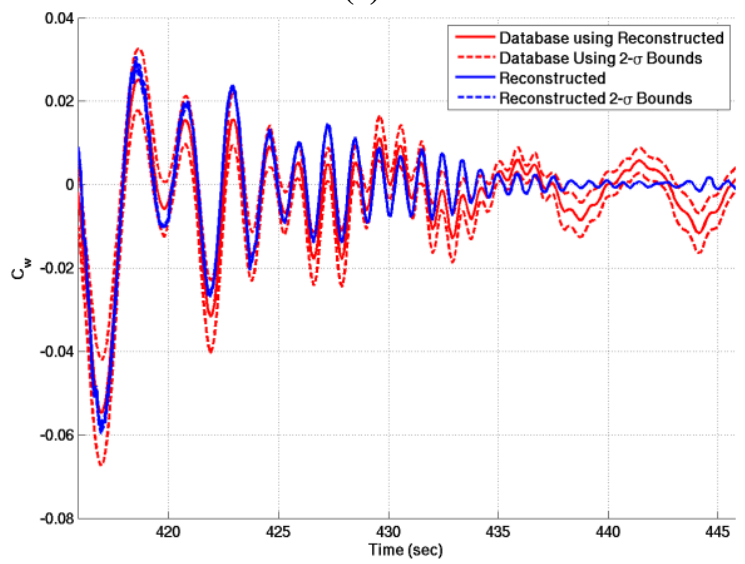

(e)

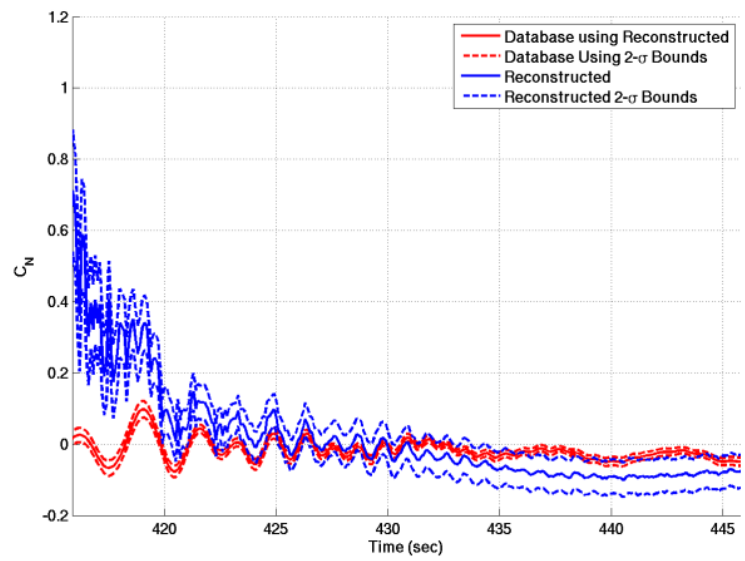

(b)

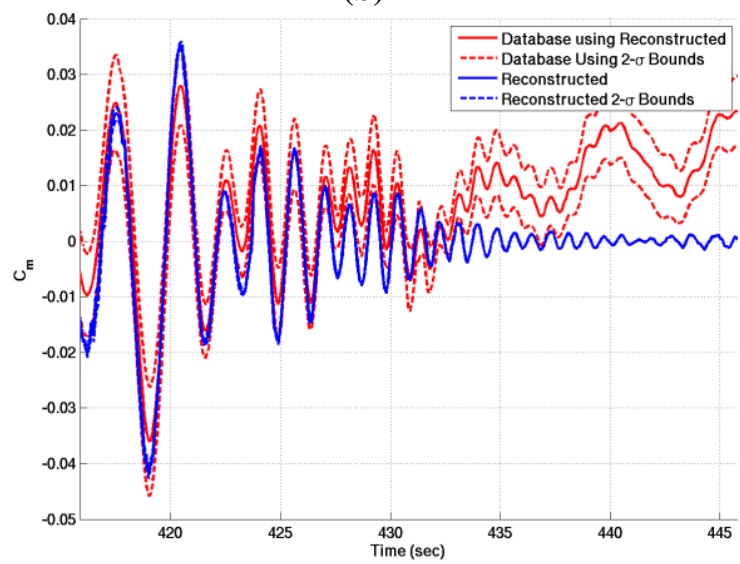

(d)

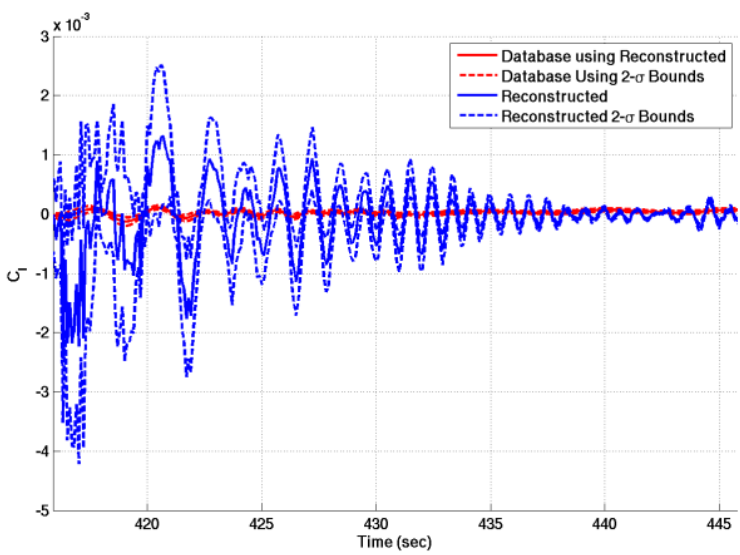

(f)

Figure 6. Reconstructed aerodynamic coefficients compared to pre-flight database.

American Institute of Aeronautics and Astronautics 092407 


\section{Post-Flight Monte-Carlo Predictions}

The reconstruction results compare better to the post-flight Monte Carlo, in which the vehicle state at separation from the launch vehicle and timeline for inflation are updated. Plots of these results can be seen in Figure 5. Due to the ballistic nature of the trajectory prior to atmospheric interface, updating the initial conditions aligns the nominal, 50 percentile values and the reconstruction results as well as reducing the $2 \sigma$ bounds for most parameters. The total angle of attack bounds increase relative to the pre-flight Monte Carlo, but this is expected due to the uncertainty in the NSROC solution and atmospheric parameters, especially winds, at higher altitudes.

IRVE-II's reconstructed total angle of attack, both wind relative and inertial, compares well with the 50 percentile results of the post-flight Monte Carlo. However, between 435 and 445 a low frequency oscillation is seen that is not predicted by the simulation near peak dynamic pressure. The cause of this effect is not fully understood, but may be due to aeroshell deflection as discussed earlier. The reconstructed trajectory still exhibits higher than predicted dynamic pressure, with a value $8 \%$ higher than predicted at peak.

\section{Comparison to Aerodynamic Database}

NewSTEP also produces estimates and error bounds for aerodynamic coefficients in addition to the trajectory states. Input mass properties and sensed accelerations are used to calculate forces, which are then converted into coefficients using the atmospheric state and input reference area and length. The aeroshell is assumed to have a circular reference area with a diameter of $2.93 \mathrm{~m}$ based on a pre-flight laser scan. A similar process is used to produce the moment coefficients.

By inputting the reconstructed trajectory states into the pre-flight aerodynamic database, predictions of the aerodynamic coefficients for the flown trajectory are generated. Estimated values and uncertainty bounds are calculated for axial, normal and side force coefficients and pitching, yawing and rolling moment coefficients using the reconstructed Mach, angle of attack and angle of sideslip. The uncertainties in these parameters are combined with the inherit uncertainty of the aerodynamic database to create $2 \sigma$ bounds on the predicted aerodynamic coefficients.

Comparisons between the NewSTEP reconstructed aerodynamic coefficients and the aerodynamic database computed coefficients are shown in Figure 6. The flight axial force coefficient is lower than that predicted by the aerodynamic database and exhibits oscillations between 430 and 445 seconds not seen in the database predictions. The normal and side force coefficients show similar trends albeit with differing magnitudes.

The reconstructed pitching moment coefficient aligns with aerodynamic database until 430 seconds, showing similar oscillations and amplitudes. After this point the database predicts a higher value during the peak pressure pulse. The reconstructed rolling moment coefficient shows similar trends to the predicted values, but while the magnitude of the coefficient is small, it is much larger than predicted.

\section{Alternative Observations}

Alternative data sources and/or methods are used to verify the reconstructed trajectory. This includes using accelerations and the pre-flight aerodynamic model to calculate an axial force coefficient as well as using video to estimate vehicle attitude. These two alternative observations are discussed further in the following paragraphs.

\section{Axial Force Coefficient}

The accelerometer based estimate of $C_{A}$ is shown relative to the pre-flight aerodynamic database assuming the BET Mach and total angle of attack in Figure 7. Early in the entry, the measured $C_{A}$ is consistently lower than the $2 \sigma$ low pre-flight database prediction. However, around 430 seconds, the measured $C_{A}$ begins to converge with the preflight prediction. At peak pressure, the difference between the measured and pre-flight $C_{A}$ is roughly $7 \%$. This corresponds well to the differences seen in dynamic pressure.

Although the discrepancy at peak pressure is nearly within $2 \sigma$ uncertainty bounds, it is likely that the flexibility of the inflatable during the pressure pulse is impacting drag performance. Either a reduction in the effective cone angle or an increase in the effective total angle of attack of the

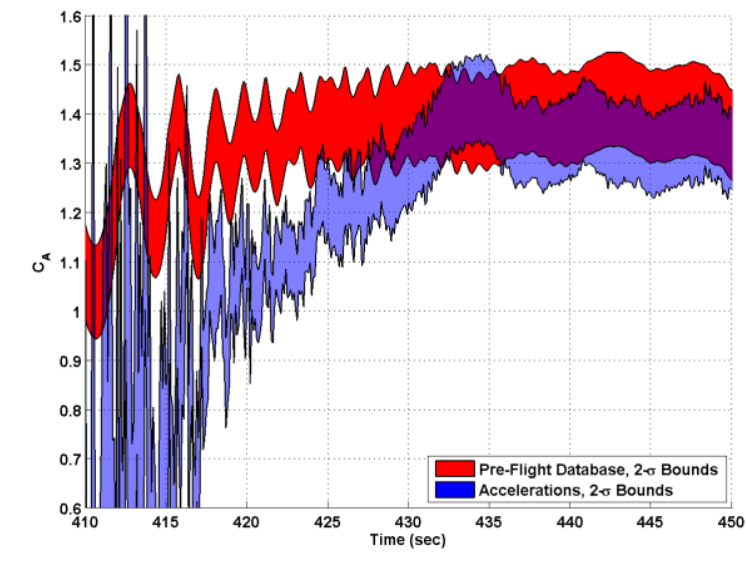

Figure 7. $C_{A}$ calculated from accelerations compared to the pre-flight aerodynamic database prediction. 
aeroshell of roughly 5 to 10 degrees could explain the reduced axial force coefficient.

2. Attitude from Video

The quality of the in-flight video presented another means of observing IRVE-II entry trajectory, specifically the vehicle's attitude. By positioning 3D CAD models in the NASA Synergistic Engineering Environment (SEE) tool according to the BET latitude, longitude, and altitude, an estimate of attitude is determined through comparison to video. More specifically, the NSROC attitude solution is used to initialize the orientation of the vehicle in SEE and adjustments are made to IRVE-II's attitude to match flight images so as to ascertain an estimate of the potential error in the NSROC solution. Analysis focused on the time between inflation and the beginning of the experiment

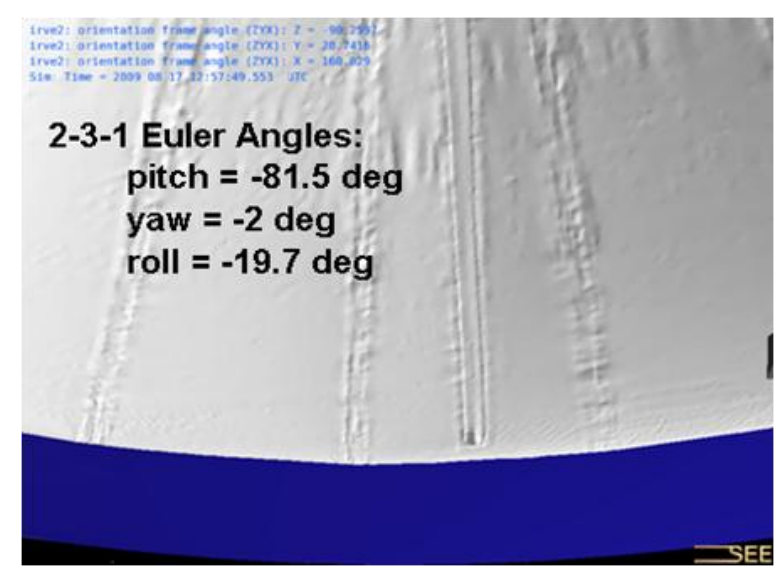

(a)

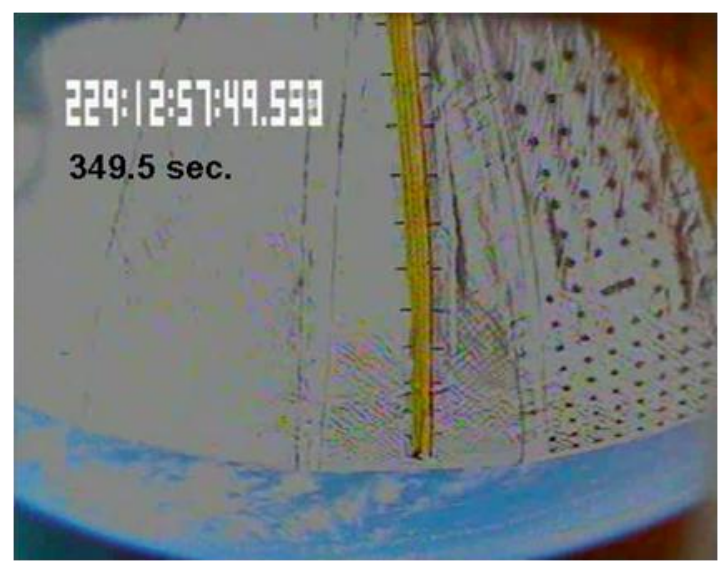

(b)

Figure 8. Flight video compared to SEE visualization at $349.5 \mathrm{sec}$.

assuming a rigid aeroshell. During this time, accelerations are low and the NewSTEP solution shows larger errors. Since this is also prior to the pressure pulse, the assumption of inflatable aeroshell rigidity is sound.

An example result of this analysis is provided in Figure 8. At this time point, the CAD models and the flight video compare well with no adjustments. Note that the CAD-based image does not duplicate the fish-bowl perspective provided by the flight cameras. Despite this shortcoming, it is evident that the same amount of the Earth is visible beyond the edge of the aeroshell and the significant seams on the backside of the aeroshell are aligned. At other time points, adjustments of 5 to 10 degrees in pitch and yaw are required to improve comparisons. Larger differences are seen in roll angle. Much of the adjustment can be explained by the potential for small phase error in the NSROC solution, a 10 degree error in roll corresponds to roughly a 0.2 second phase error.

\section{E. Other Flight Events}

Other flight events of interest include the sounding rocket separation and aeroshell deployment events. An assessment of these events, relative to pre-flight analysis, is provided in this section.

\section{Payload Separation}

Payload separation was a considerable pre-flight concern on IRVE-II given the IRVE-I flight mishap during this flight event. Two key changes to the separation

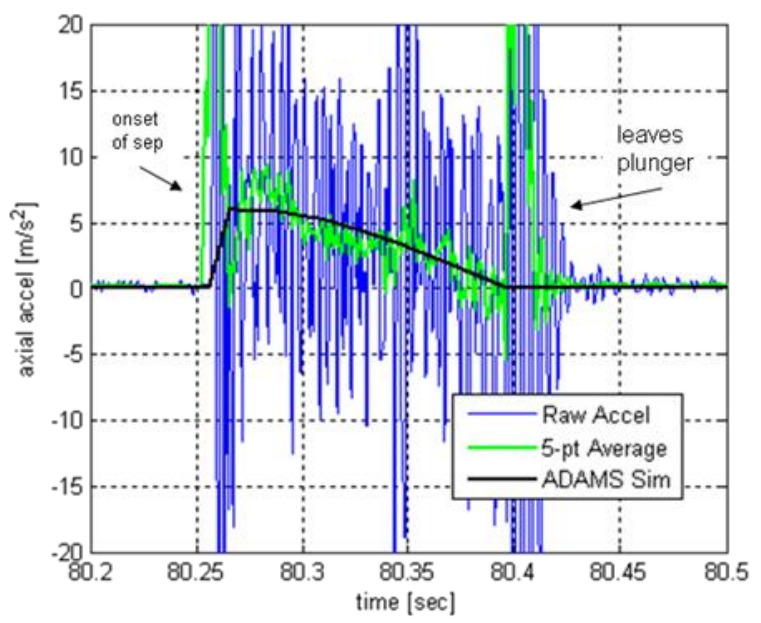

(a)

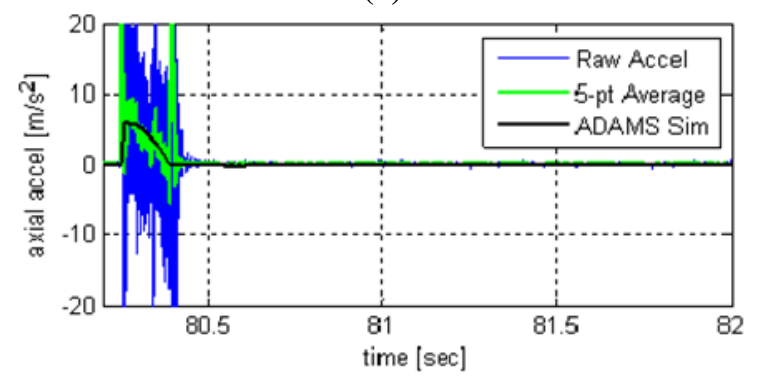

(b)

Figure 9. Axial acceleration during payload shroud separation.

American Institute of Aeronautics and Astronautics 092407 
strategy for IRVE-II were made to improve the probability of success. These included the move to a different launch vehicle configuration and a larger payload shroud. The larger shroud affords more clearance between the payload shroud and IRVE-II. The other change involves the shroud separation ejector springs. The four-spring array used on IRVE-I was modified to a parallel, plunger arrangement, seen by WFF to impart less tip-off rate.

The predicted performance of the shroud ejector springs is illustrated in Figure 9 relative to flight-measured axial acceleration. The blue trace is the raw axial accelerometer values from IRVE-II. The green line represents an 11point, centered average of these raw measurements, employed to remove high frequency noise. This eleven point average imparts negligible gain loss at the frequencies of interest. After filtering, the black line taken from pre-flight separation simulation provides an excellent match to the measured accelerations. Further, as illustrated in Figure 9 (b) there is no evidence of re-contact between IRVE-II and the payload shroud.

Pre-flight analysis of separation focused on identifying the worst case separation disturbance, ensuring that IRVE-II entry performance was robust to such a disturbance. This analysis modeled worst case conditions including an initial tip-off rate following the jettison of the $2^{\text {nd }}$ stage motor, ejector spring misalignments, contact stiffness and damping, and mass imbalances. The worst case, pre-flight prediction of total tip-off rate due to separation was 6.7 $\mathrm{deg} / \mathrm{sec}$ and the worst case angular momentum shift due to separation was estimated to be 7.5 degrees.

Using IRVE-II rate sensor measurements, adjusted for bias, the tip-off rates during separation are estimated. Figure 10 illustrates IRVE-II tip-off rate including the raw measurements (blue) and an average (green). Although the tip-off rate increases due to the jettison of the $2^{\text {nd }}$ stage motor at 70 seconds, it is well below the worst case value of $3.57 \mathrm{deg} / \mathrm{sec}$ modeled pre-flight. At 80 seconds, the payload skirt is separated from the main payload. Here there is a small change in tip-off rate, which is further evidence of a clean shroud separation. At 90 seconds, IRVE-II is separated from the telemetry module (TM) and nosecone assembly. The tip-off rate drops to $1.2 \mathrm{deg} / \mathrm{sec}$, which is well below the $6.7 \mathrm{deg} / \mathrm{sec}$ seen in worst case analysis.

The separation induced attitude upset was also of concern pre-flight, as it directly impacts the entry angle of attack. This impact was captured in the initial tip-off rate, assumed to be at worst $3.57 \mathrm{deg} / \mathrm{sec}$. Worst case simulations predicted an initial nutation of 6.5 degrees and a final shift in angular momentum of 7.5 degrees. This worst case shift occurs when the separation of IRVE-II from the TM/Nosecone assembly happens at the peak of its coning motion.

The NSROC attitude solutions of the sounding rocket and IRVE-II provide a means of comparing IRVE-II attitude during separation to pre-flight predictions as seen in Figure 11. Qualitatively, the dynamics are strikingly similar. Quantitatively, the payload is initially coning with a nutation angle of roughly 2.6 degrees about an angular momentum vector that is 9.5 degrees away from vertical (green ' $\mathrm{X}$ '). Following the separation events, IRVE-II has a nutation angle less than 1 degree and an angular momentum vector that is 10.5 degrees from vertical. Therefore, the total angular momentum shift due to the separation is 1 degree from vertical, far below the 7.5 degrees worst case prediction.

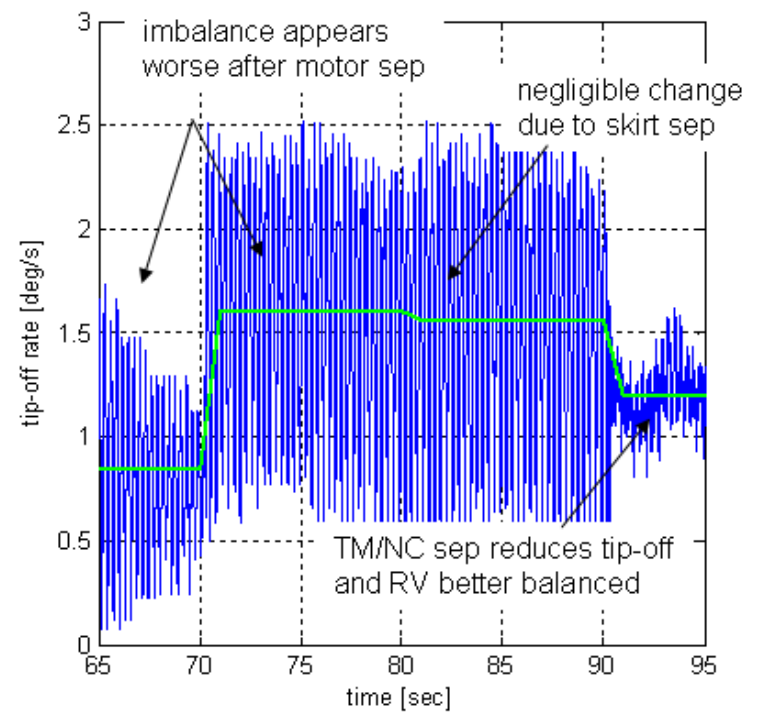

Figure 10. RV lateral body rates during the separation events.

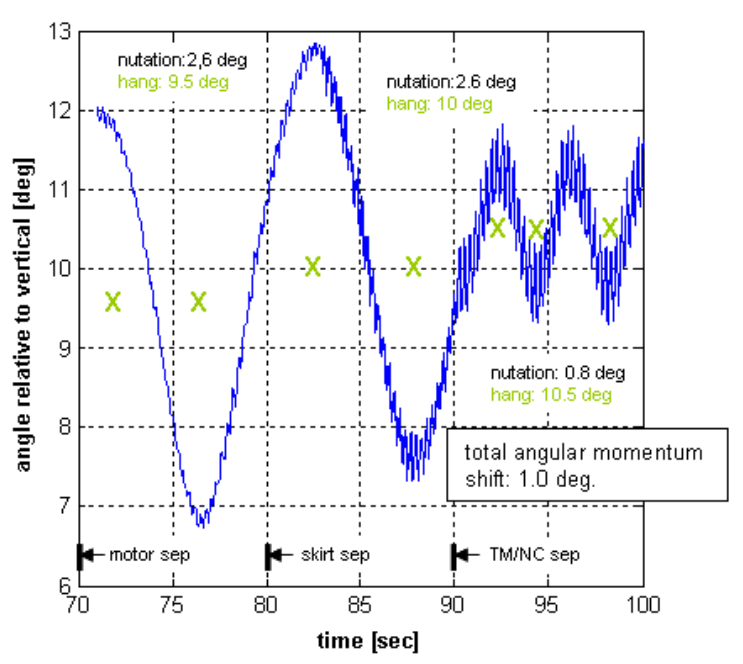

Figure 11. Attitude relative to vertical during separation, pre-flight simulations.

10

American Institute of Aeronautics and Astronautics 092407 


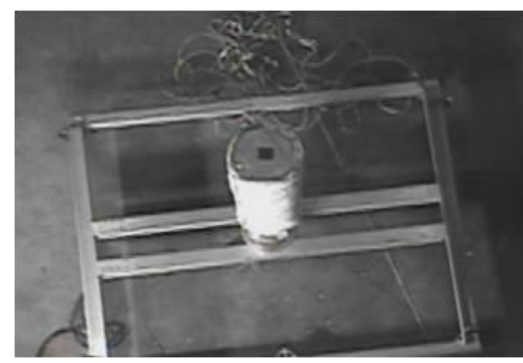

(a) Stowed

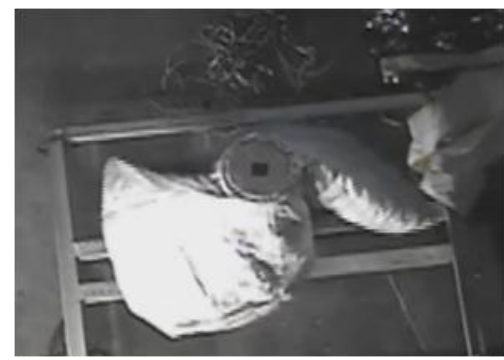

(b) Intermediate

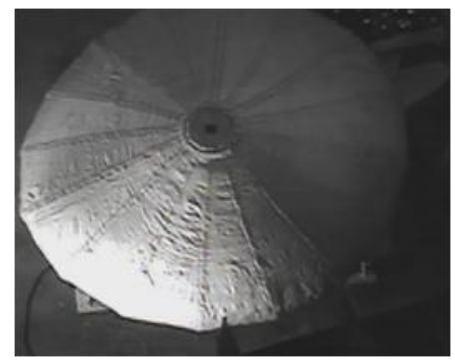

(c) Deployed

Figure 12. Evolution of the aeroshell deployment during ground testing.

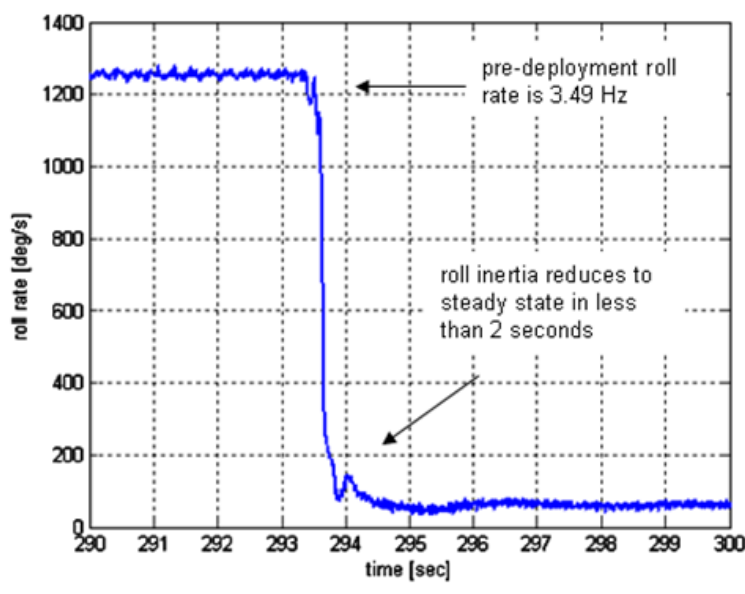

Figure 13. Roll rate during deployment.

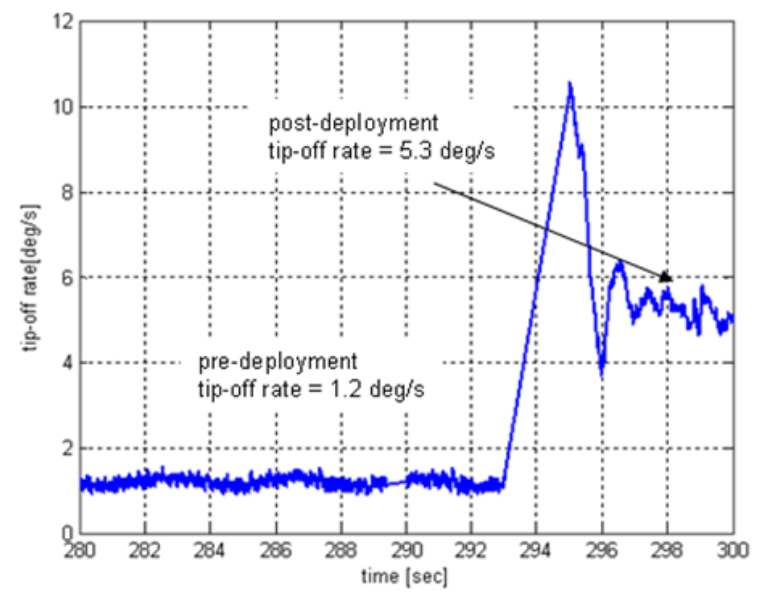

Figure 14. Tip-off rate during deployment.

\section{Deployment}

Inflation of the aeroshell is another event of significant interest. Due to the high level of complexity associated with this event, the pre-flight strategy was to model deployment as a disturbance event. A wide range of disturbance torques were evaluated in the IRVE-II POST2 simulation to ensure the simulation had a high level of robustness in terms of the resulting entry performance, most importantly entry total angle of attack. In an attempt to put these disturbance torques into some context, a simulation was also conducted that emulated potential mass imbalance during the deployment. This mass imbalance was modeled after the evolution in shape of the aeroshell seen during full-scale deployment ground testing in the NASA Langley Research Center 16-m vacuum sphere. Images taken during this test can be seen in Figure 12. Since the vehicle is not spinning in the ground test it was unlikely this evolution would be duplicated in flight. However, it does provide some insight into bounding the problem. When simulating the mass properties of this shape evolution, the resulting tip-off rate equated to roughly a $12 \mathrm{~N}-\mathrm{m}-\mathrm{s}$ disturbance.

In comparison to pre-flight analysis, the flight deployment occurred over a very short duration. As seen in the roll rate time history plotted in Figure 13. IRVE-II reaches its full roll inertia in less than 2 seconds. The effective tip-off rate of the deployment, seen in Figure 14, is $4.1 \mathrm{deg} / \mathrm{sec}$. When cross-referenced with the pre-flight simulation this equates to a flight disturbance between 0 and $4 \mathrm{~N} \cdot \mathrm{m} \cdot \mathrm{s}$.

\section{Conclusion}

IRVE-II successfully demonstrated an inflatable aerodynamic decelerator and flight trajectories have been reconstructed to fidelity sufficient to assess overall project objectives related to flight dynamics. Overall, IRVE-II flight dynamics are in line with expectations. Accounting for the high boost velocity, the re-entry trajectory followed a nearly nominal profile indicating the expected drag performance. The re-entry vehicle appeared stable throughout the flight, until the aeroshell lost sufficient pressure to remain inflated. Based on these findings, the methodologies and design principals employed in IRVE-II flight dynamics and aerodynamics analyses are corroborated.

Additional work, however, is recommended to further reconcile differences between pre-flight and post-flight predictions of body-axis accelerations and reconstructed aerodynamic coefficients. Collaboration with structures and 
photogrammetric analysis is needed to understand and account for the structural dynamics seen during the entry pressure pulse, particularly deformation and deflection of the inflatable article.

Lessons learned through the IRVE-II trajectory reconstruction have been provided to the proposed IRVE-3 project. These lessons include ensuring required accuracy of post-flight data products drives the specification of flight instrumentation, improving the quality of the instrumentation available and supporting redundancy of reconstruction approaches. Adding instrumentation to directly quantify any inflatable deflection and deformation has also been recommended.

\section{References}

${ }^{1}$ Hughes, S. J., Dillman, R. A., Starr, B. R., Stephan, R. A., Lindell, M. C., Player, C. J., and Cheatwood, F. M., "Inflatable Re-entry Vehicle Experiment (IRVE) Design Overview," AIAA Paper 2005-1636-292, May 2005.

${ }^{2}$ Karlgaard, C.D., Tartabini, P.V., Blanchard, R.C., Kirsch, M., and Toniolo, M.D., "Hyper-X Post-Flight Trajectory Reconstruction," Journal of Spacecraft and Rockets, Vol. 43, No. 1, 2006, pp. 105-115. 